\title{
ANOTHER LOOK AT THE EASTERN BANDED STRUCTURE: A STELLAR DEBRIS STREAM AND A POSSIBLE PROGENITOR
}

\author{
C. J. GRILlmair \\ Spitzer Science Center, 1200 E. California Blvd., Pasadena, CA 91125, USA; carl@ipac.caltech.edu \\ Received 2011 June 24; accepted 2011 June 24; published 2011 August 16
}

\begin{abstract}
Using the Sloan Digital Sky Survey Data Release 7, we re-examine the Eastern Banded Structure (EBS), a stellar debris stream first discovered in Data Release 5 and more recently detected in velocity space by Schlaufman et al. The visible portion of the stream is $18^{\circ}$ long, lying roughly in the Galactic Anticenter direction and extending from Hydra to Cancer. At an estimated distance of $9.7 \mathrm{kpc}$, the stream is $\approx 170 \mathrm{pc}$ across on the sky. The curvature of the stream implies a fairly eccentric box orbit that passes close to both the Galactic center and to the Sun, making it dynamically distinct from the nearby Monoceros, Anticenter, and GD-1 streams. Within the stream is a relatively strong, $2^{\circ}$-wide concentration of stars with a very similar color-magnitude distribution that we designate Hydra I. Given its prominence within the stream and its unusual morphology, we suggest that Hydra I is the last vestige of EBS's progenitor, possibly already unbound or in the final throes of tidal dissolution. Though both Hydra I and the EBS have a relatively high-velocity dispersion, given the comparatively narrow width of the stream and the high frequency of encounters with the bulge and massive constituents of the disk that such an eccentric orbit would entail, we suggest that the progenitor was likely a globular cluster and that both it and the stream have undergone significant heating over time.
\end{abstract}

Key words: Galaxy: halo - Galaxy: structure - globular clusters: general

Online-only material: color figure

\section{INTRODUCTION}

At least 14 stellar debris streams in the Galactic halo have now been identified in photometric surveys (see Grillmair 2010 for a review). A similar number of dynamically cold substructures have been detected in velocity space (Helmi et al. 1999; Smith et al. 2009; Schlaufman et al. 2009; Williams et al. 2011). Each of these streams is interesting as a partial record of the accretion history of our Galaxy. However, and perhaps more importantly, these streams can also serve as very sensitive probes of the Galactic potential (Law et al. 2009; Koposov et al. 2010). Globular cluster streams are particularly important in this respect as they are dynamically very cold (Combes et al. 1999; Odenkirchen et al. 2009; Willett et al. 2009). A large sample of streams will eventually enable us to constrain the distribution of dark matter in the halo in a detailed and self-consistent manner. Enlarging the sample of known streams will also increase the probability that we may detect unmistakable signs of perturbations by dark matter subhalos (Murali \& Dubinski 1999; Carlberg 2009; Yoon et al. 2010).

In this paper we re-examine the Eastern Banded Structure (EBS) first detected by Grillmair (2006b) using the more complete coverage available in the Sloan Digital Sky Survey (SDSS) Data Release 7 (DR7; Abazajian et al. 2009). We briefly describe our analysis in Section 2. We characterize the EBS and a possible progenitor in Section 3, and we put preliminary constraints on the orbit in Section 3.2. We make concluding remarks Section 4.

\section{DATA ANALYSIS}

Data comprising $g, r$, and $i$ photometry for $7 \times 10^{7}$ stars in the region $108^{\circ}<\alpha<270^{\circ}$ and $-4^{\circ}<\delta<65^{\circ}$ were extracted from the SDSS DR7 database using the SDSS CasJobs query system. The data were analyzed using the matched filter technique described by Rockosi et al. (2002) and Grillmair (2009). Applied in the color-magnitude domain, the matched filter is a means by which we can optimally differentiate between halo streams and the foreground disk population.

In this paper, we use filters based on the Padova database of theoretical stellar isochrones (Marigo et al. 2008; Girardi et al. 2010). The advantages of using theoretical isochrones include the ability to explore a wider range of age and metallicity than is available among the globular clusters within the SDSS footprint, as well as the ability to extend the filters to very faint absolute magnitudes (useful for examining very nearby structures). These isochrones were combined with the deep luminosity function of $\Omega$ Cen measured by de Marchi (1999) to generate appropriate filters. We used all stars with $15<g<22$, and we dereddened the SDSS photometry as a function of position on the sky using the prescription of Schlafly \& Finkbeiner (2011) applied to the $E(B-V)$ maps of Schlegel et al. (1998). The field star distribution was sampled using roughly half the Sloan survey area. We applied the filters to the entire survey area, and the resulting weighted star counts were summed by location on the sky to produce two-dimensional, filtered surface density maps.

In Figure 1, we show the filtered star count distribution using a filter based on an isochrone with $Z=0.0003$ and an age of $13 \mathrm{Gyr}$, shifted in magnitude so as to provide optimal contrast for display purposes for stellar populations at a distance of $9.7 \mathrm{kpc}$. The surface density map was generated by averaging the weights of each star based on its distance from the $g-r$ and $g-i$ color-magnitude loci. The surface densities have been binned to a pixel size of 0.1 and smoothed using a Gaussian kernel with $\sigma=0.2$.

\section{DISCUSSION}

The region shown in Figure 1 is a complex of streams overlaid on a rapidly rising population of foreground disk stars. 


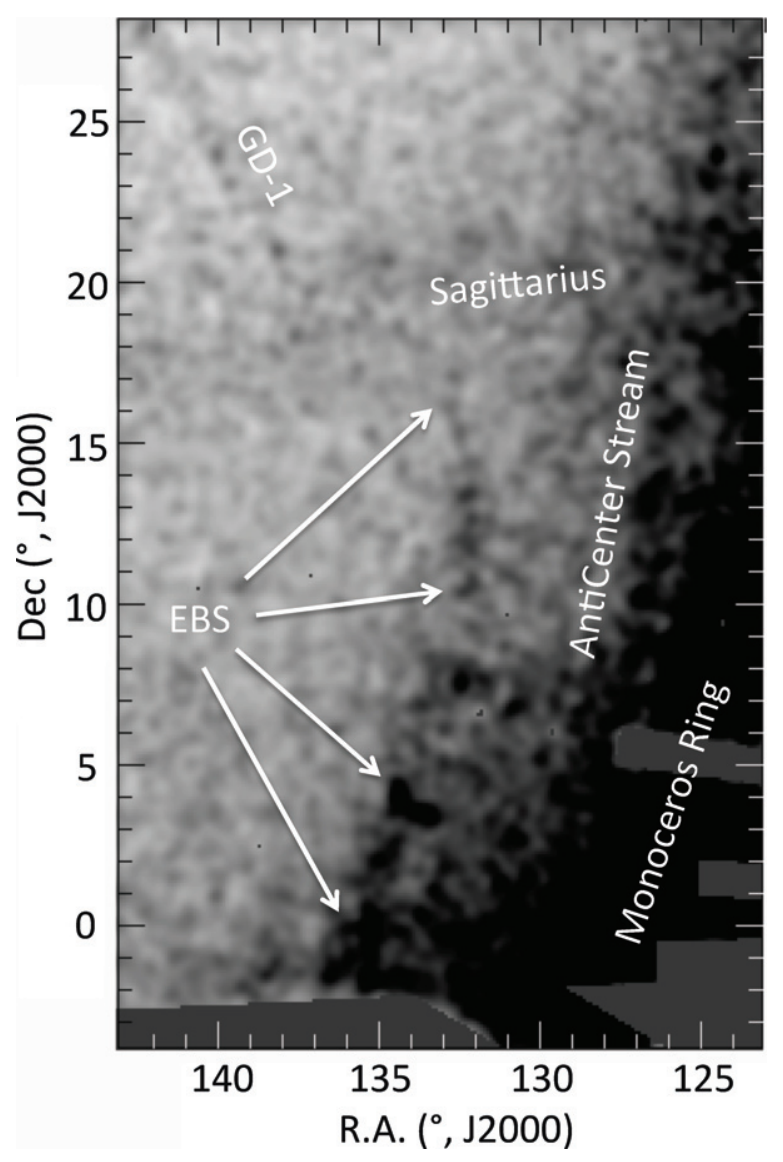

Figure 1. Filtered surface density map of the southwest corner of the SDSS DR7 footprint. The stretch is logarithmic, and darker areas indicate higher surface densities. The map is the result of a filter based on a Padova isochrone with [Fe/ $\mathrm{H}]=-1.8$, an age of $13 \mathrm{Gyr}$, and shifted to a distance of $9.7 \mathrm{kpc}$. The results have been smoothed with a Gaussian kernel of width 0.2 , and no background subtraction has been applied. Other known streams are indicated.

Visible to varying degrees (due to the $(m-M)_{0}=14.83 \mathrm{mag}$ shift of the filter) are five well-known features, namely, the
Sagittarius stream (Belokurov et al. 2006a), the Monoceros Ring (Newberg et al. 2002; Yanny et al. 2003), the Anticenter Stream (ACS, Grillmair 2006b), GD-1 (Grillmair \& Dionatos 2006b), and the EBS (Grillmair 2006b). While the EBS was only partly revealed in Grillmair (2006b)'s DR5 analysis due to a large swath of missing data, the additional coverage in DR7 allows us to trace the EBS for some $18^{\circ}$ from the southern edge of the DR7 footprint in Hydra to an indeterminate end in Cancer. The curvature of the stream takes it to within $4^{\circ}$ of the similarly curved, southern end of the GD-1 stream, but there are clear discontinuities in position, distance, and color-magnitude distribution (CMD) that rule out any physical association between them.

Grillmair (2009) used a significance test (the " $T$-statistic") that measures the median contrast along its length between a putative stream and the surrounding field. The $T$-statistic for the EBS, comparing with the field extending the length of the stream and $15^{\circ}$ to the east, is shown in Figure 2. The stream is clearly not due to random fluctuations in the field; the filtered stream signal is $\sim 28 \times$ larger than the rms measured using identically sampled, neighboring field stars. A Gaussian that matches the integrated, lateral profile of the stream has a full width at halfmaximum (FWHM) of 1.0. At a distance of $9.7 \mathrm{kpc}$ (see below), this corresponds to a spatial extent perpendicular to our line of sight of $170 \mathrm{pc}$. This is roughly twice as broad as typical, presumed globular cluster streams (Odenkirchen et al. 2003; Belokurov et al. 2006a; Grillmair \& Johnson 2006; Grillmair \& Dionatos 2006a, 2006b; Grillmair 2009), but considerably narrower than the $>1 \mathrm{kpc}$ widths associated with presumed dwarf galaxy streams (Majewski et al. 2003; Martinez-Delgado et al. 2004; Grillmair 2006a, 2006b; Belokurov et al. 2006b, 2007; Grillmair 2009). In the absence of heating effects due to a rather eccentric orbit (see below), this would suggest a progenitor that was significantly more massive than the globular clusters believed to be responsible for the currently known cold streams.

Figure 3 shows CMDs, dereddened as prescribed by Schlafly \& Finkbeiner (2011) using the $E(B-V)$ maps of Schlegel

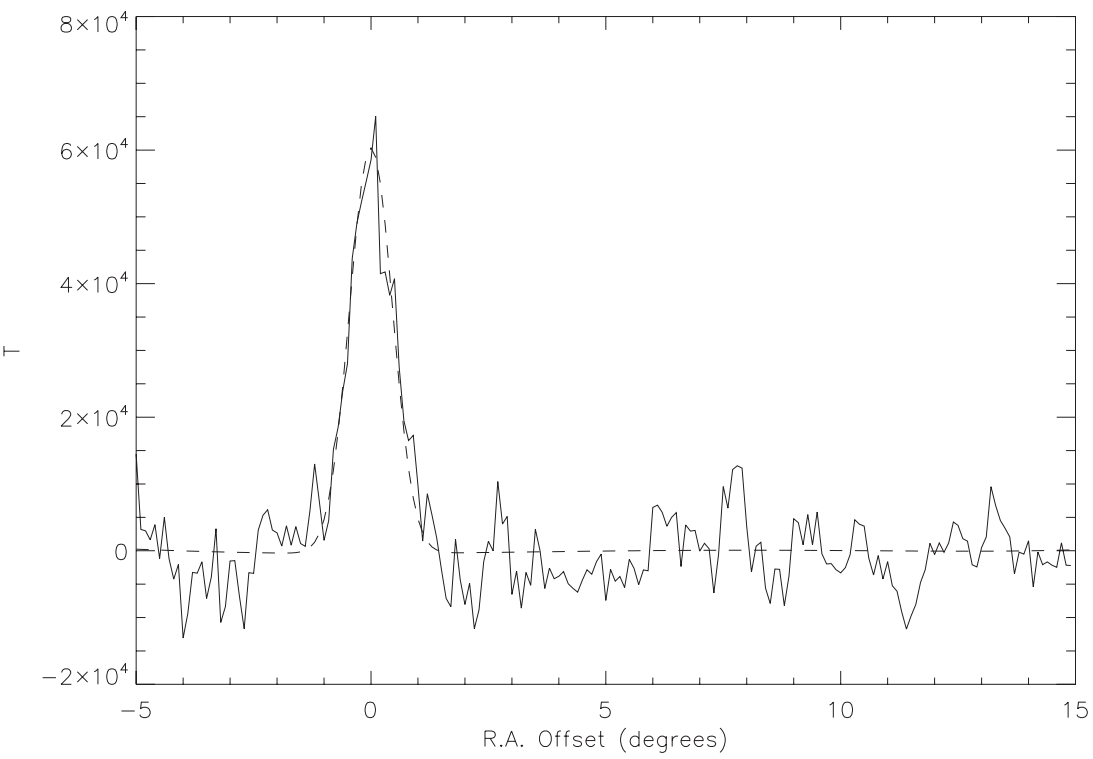

Figure 2. The "T" statistic (Grillmair 2009), showing the background-subtracted, median filtered signal over five, $3^{\circ}$-long segments, integrated over a width of one degree, as a function of lateral offset from the stream. The peak value is 28 times larger than the rms measured for the identically sampled region between $2^{\circ}$ and $15^{\circ}$ east of the stream, indicating a very low probability that the stream could be due to random fluctuations in the field. The dashed line shows a Gaussian with an FWHM of 1.0 , which we take as a measure of the average breadth of the stream. 


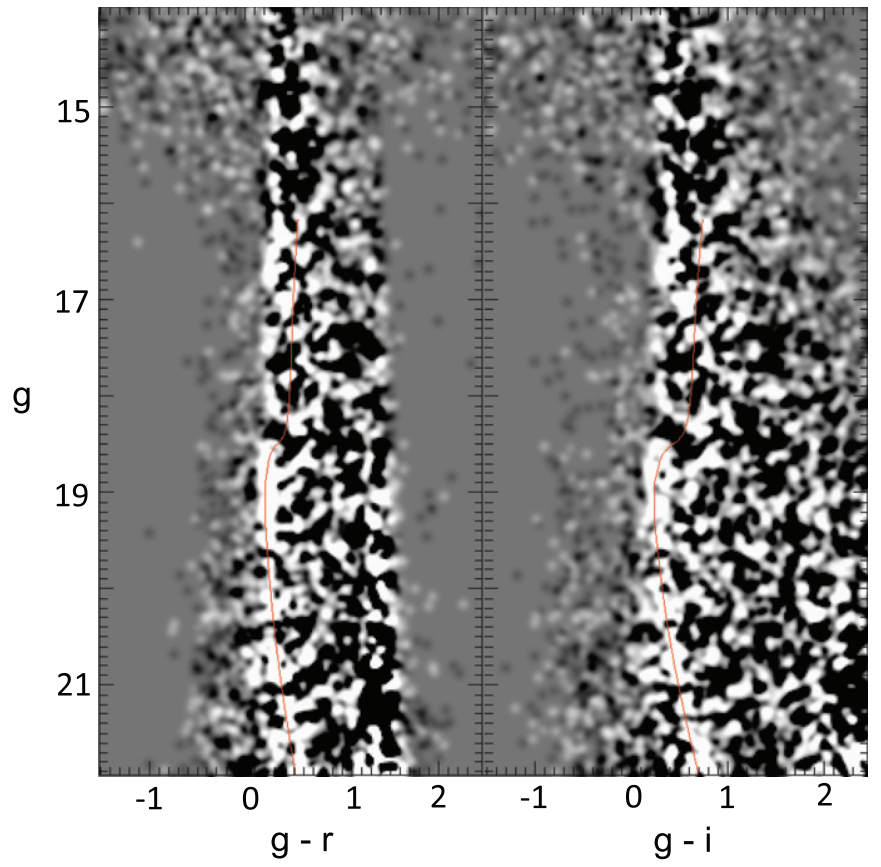

Figure 3. Hess diagrams of the stars lying within $1^{\circ}$ of the centerline of the EBS Padova isochrones with $[\mathrm{Fe} / \mathrm{H}]=-1.8$, age $13 \mathrm{Gyr}$, and shifted to a distance of $9.7 \mathrm{kpc}$ are overplotted. Lighter areas indicate higher surface densities.

(A color version of this figure is available in the online journal.)

et al. (1998), of stars lying within $1^{\circ}$ of the centerline of the EBS, after subtraction of the CMD of stars lying between 2.4 and 4.0 both east and west of the stream. Isochrones with $Z=$ $0.0003([\mathrm{Fe} / \mathrm{H}] \approx-1.8)$ and an age of 13 Gyr evidently match the turnoff and main-sequence colors reasonably well. We infer that the progenitor of the stream was old and metal poor.

Following Grillmair \& Dionatos (2006b), we shift the main sequence used to construct our filter both brightward and faintward to estimate the stream's distance. To avoid issues related to a possible difference in age between our adopted isochrone and the stream stars, we use only the portion of the filter with $19.5<g<22.0$, where the bright cutoff is $0.3 \mathrm{mag}$ below the main-sequence turnoff. We find that the strength of the southern half of the stream peaks at a distance modulus of $14.9 \pm 0.2 \mathrm{mag}$, while the northern half of the stream peaks at $14.8 \pm 0.3 \mathrm{mag}$. This puts the southern end of the stream at a Sun-centric distance of $9.7 \pm 0.9 \mathrm{kpc}$, while the northern end is at $9.4 \pm 1.4 \mathrm{kpc}$. The portion of the stream visible in Figure 1 is evidently almost perpendicular to our line of sight.

Integrating the background-subtracted, unfiltered counts of stars within $3 \sigma$ of the $Z=0.0003$ isochrone along the length of the stream and over a width of 1.5 , we find the total number of stars in the discernible stream to be $530 \pm 230$. The large uncertainty simply reflects the Poisson statistics of the very high background $(\approx 33,000$ field stars in the same region of color and configuration space). Figure 4 shows a background-subtracted, longitudinal profile of the filtered star counts, normalized to yield an integrated total of 530 stars. For stars with $g<22$ and a stream width of 1.0 , the average surface density is $30 \pm 13$ stars $\mathrm{deg}^{-2}$, with a peak of over $100 \mathrm{stars} \mathrm{deg}^{-2}$. Like the Pal 5 and GD-1 streams, the EBS profile shows interesting peaks and troughs, fairly regularly spaced with a separation of $4.0 \pm 0.2$. While the uncertainties are large, the clumps and gaps that give rise to these features appear quite obvious in Figure 1. The regular spacing may suggest an origin tied to the orbit of the stream, perhaps a result of episodic stripping (e.g., major stripping pulses at the perigalacticon of a highly eccentric orbit (Grillmair 1992; Johnston et al. 1995)). Alternatively, the undulations may be due to scattering by encounters with massive objects in the disk or halo (Murali \& Dubinski 1999; Yoon et al. 2010).

Schlaufman et al. (2009) recently detected a number of cold halo substructures in velocity space ("ECHOS") using SEGUE data. With a plate center at (R.A., decl.) $=(132.6,6.1)$, their B-7/PCI-8/PCII-20 detection overlaps the EBS (Figure 5) and the estimated distance of $10 \mathrm{kpc}$ is almost identical to what we find for the stream. We have examined this region using isochrone filters with $[\mathrm{Fe} / \mathrm{H}]$ ranging from -2.2 to 0.0 and find no evidence for other cold substructures at this distance.

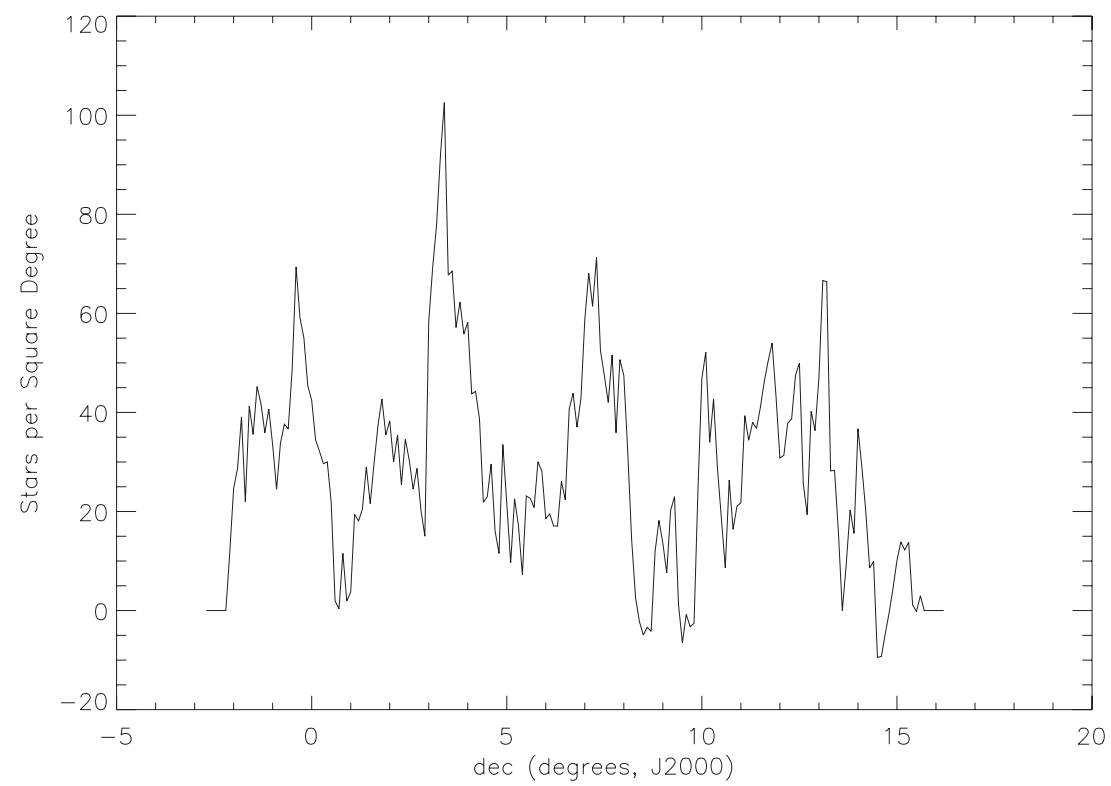

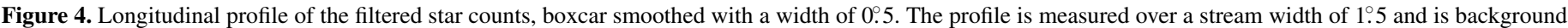

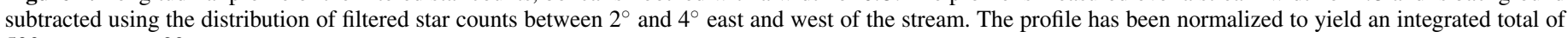
530 stars to $g=22$. 


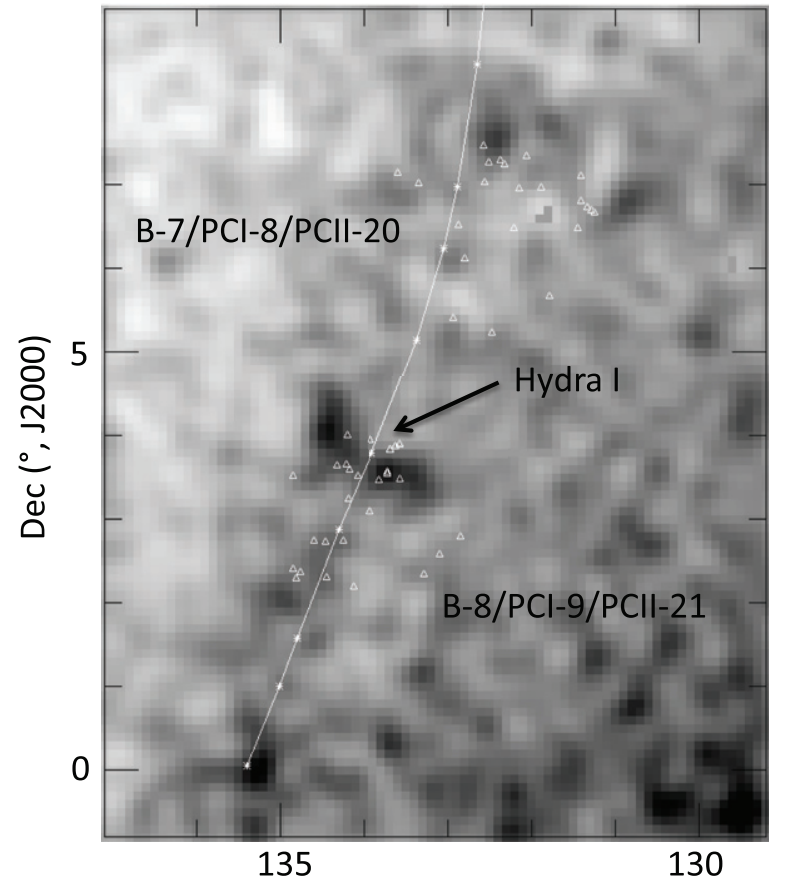

R.A. $\left({ }^{\circ}, \mathrm{J} 2000\right)$

Figure 5. Expanded, lower-contrast view of the southern portion of the EBS, showing the positions of the stars making up Schlaufman et al. (2009)'s B-7/PCI-8/PCII-20 and B-8/PCI-9/PCII-21 ECHOS detections (K. C. Schlaufman (2011, private communication)). The asterisks connected by lines show the normal points used to define the path of the stream for fitting purposes.

We conclude that B-7/PCI-8/PCII-20 is most likely sampling stars in the EBS stream.

Schlaufman et al. (2009) find a mean radial velocity for B-7/PCI-8/PCII-20 of $71 \mathrm{~km} \mathrm{~s}^{-1}$ and a dispersion of $13 \mathrm{~km} \mathrm{~s}^{-1}$. This dispersion is significantly larger than that measured for known and presumed globular cluster streams (Odenkirchen et al. 2009; Willett et al. 2009; Koposov et al. 2010). On the other hand, it is quite similar to measurements of presumed dwarf galaxy streams (Grillmair et al. 2008; Carlin et al. 2010; Newberg et al. 2010). Combining the width of the stream with its large apparent velocity dispersion, we might infer that the stream's progenitor was substantially more massive than Pal 5 or the globular clusters that produced GD-1, Acheron, Cocytos, or Lethe. On the other hand, the stream is neither as broad nor as populous as streams associated with classical dwarf galaxies like Sagittarius or the progenitor of the Orphan Stream. The high-velocity dispersion may be partly due to non-EBS stars in the sample, or it may be due to heating of the progenitor by disk or perigalactic shocking prior to the stripping of these stars. It may also be due to significant heating by encounters with either dark matter subhalos (Carlberg 2009) or massive structures (e.g., giant molecular clouds) in the disk. Another possibility is that the EBS may be the remnant of an ultrafaint dwarf galaxy (Willman et al. 2005; Grillmair 2006a; Zucker et al. 2006a, 2006b), though as we discuss below, it is difficult to imagine how such an object could have retained a dark matter envelope for any length of time.

\subsection{Hydra I: a Disrupting Progenitor?}

Figure 5 shows an expanded, lower-contrast view of the southern portion of the EBS. With a contrast maximum at the same distance $(\approx 9.7 \mathrm{kpc})$ as the EBS is an interesting and relatively compact feature at [R.A., decl.] $\approx[133.9,3.6]$. The object contains $\approx 300$ stars to $g=22$, has a filtered star count density higher than any visible portion of EBS, and appears quite distinct within the stream. There are several background galaxy clusters identified within $1^{\circ}$ of this position, but examination of the identically filtered SDSS DR7 galaxy catalog shows no significant galaxy concentration of similar size or shape. Two lesser peaks are apparent some $4^{\circ}$ north and south of this object (see also Figure 4), but we focus on this object because it is the most prominent and populous concentration, both to the eye and in the longitudinal profile.

Given its apparent location at the same distance as the stream and approximately centered within it, we infer that the feature is physically associated with EBS and we designate it Hydra I. The feature appears somewhat amorphous, with two primary concentrations extending to the north and west, respectively, for a total extent of $\approx 2^{\circ}$. At $9.7 \mathrm{kpc}$ this corresponds to a spatial extent of about $\sim 350 \mathrm{pc}$, which is far larger than any known globular cluster. Using filter shifts to estimate relative distances, we find that the maximum filtered surface densities in the two lobes of Hydra I occur within 0.1 mag of one another, indicating that Hydra I is not significantly extended along our line of sight. At $9.7 \mathrm{kpc}, 0.1 \mathrm{mag}$ corresponds to a difference in distance of $500 \mathrm{pc}$. To within the uncertainties, this is identical to the lateral extent of the object.

How real is the apparent, double-lobed morphology of Hydra I? Could the northeastern lobe be simply a chance consequence of Poisson statistics? The surface density profile of stars with $19<g<23$ in the stronger, western lobe and lying within $0.2 \mathrm{mag}$ of the $Z=0.0003$ isochrone in $g-i$ is shown in Figure 6. Fitting an elliptical $1 / r$ model to the star counts, we find ellipticity $\epsilon=0.45 \pm 0.05$, with $\theta=90 \pm 10^{\circ}$ (measured north through east), a total population out to $1^{\circ}$ of $300 \pm 10$ stars, and an overall $\chi^{2}$ of 1.2. Following Martin et al. (2008), we then determine the fractional rms deviation $\sigma_{\mathrm{sc}} /$ total of the data compared to the model. We generate 1000 Poisson realizations of the field out to $1^{\circ}$ and examine the distribution of $\left(\sigma_{\mathrm{sc}} / \text { total }\right)^{2}$. The peak of the distribution differs from 0 at the $9 \sigma$ level, indicating that the northeastern lobe is unlikely to be a statistical departure from the model. We can only speculate as to the relationship between the two lobes at this point. However, being part of a tidal stream, it seems reasonable to suppose that the two lobes are unbound, comoving tidal remnants. Deeper imaging of Hydra I is currently being acquired and will be the subject of a future contribution.

Schlaufman et al. (2009) detected a velocity overdensity among metal poor stars at [R.A., decl.] $=\left[134^{\circ}, 3^{\circ} .2\right]$ that they attributed to the Monoceros ring. Figure 5 shows that the stars making up their B-8/PCI-9/PCII-21 detection clearly sample the position of Hydra I, as well as a portion of the EBS extending to the south (which would be expected to have a nearly identical velocity). Their estimated distance of $9.7 \mathrm{kpc}$ is identical with the values we find above. Filtering this region of DR7 using isochrones with metallicities spanning the range $-2.2<$ $[\mathrm{Fe} / \mathrm{H}]<0.0$ reveals no other significant structures at this position. We conclude that Schlaufman et al. (2009) actually sampled the stars in Hydra I and the EBS. They find a mean velocity for B-8/PCI-9/PCII-21 of $85 \mathrm{~km} \mathrm{~s}^{-1}$ and a dispersion of $14.9 \mathrm{~km} \mathrm{~s}^{-1}$. The velocity dispersion is again quite high compared with globular clusters or ultrafaint galaxies, suggesting that Hydra I was either quite massive or has been significantly heated over time. Based on velocity dispersion, Schlaufman et al. (2011) have suggested that the progenitors of 


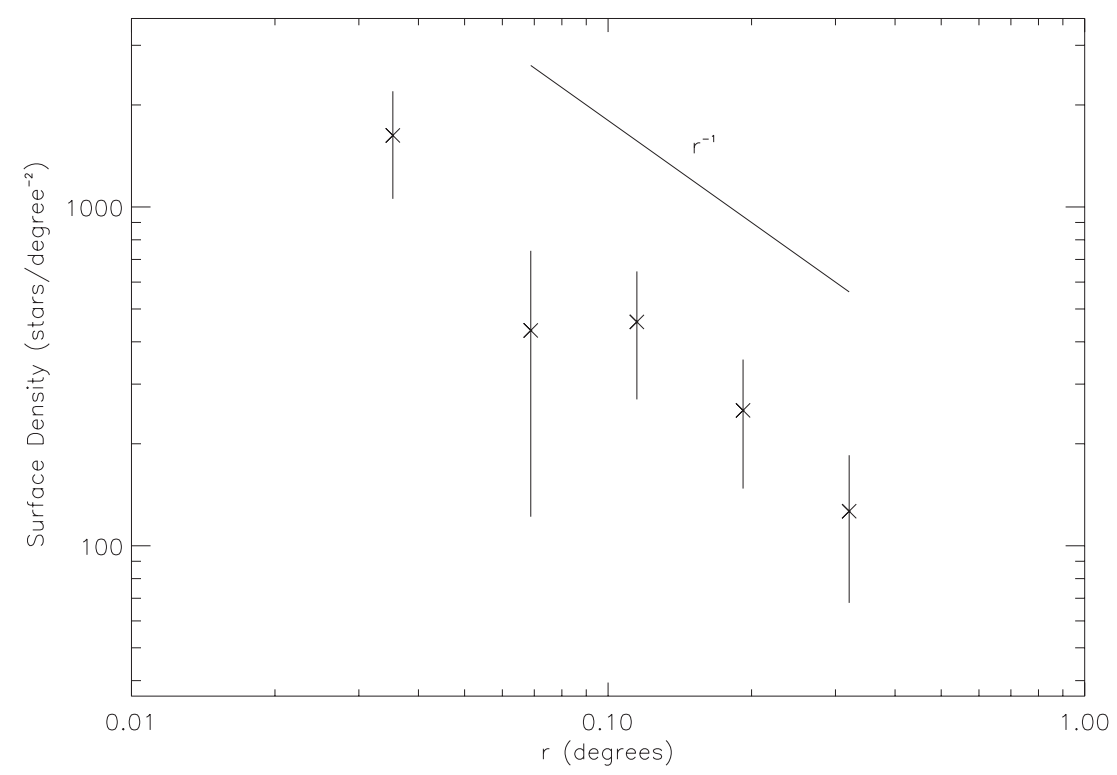

Figure 6. Surface density profile of stars in Hydra I, measured with respect to the center of the western lobe at (R.A., decl.) $=(133.450,3.465)$. Only stars with $19.0<g<23.0$ and lying within $0.2 \mathrm{mag}$ of the $Z=0.0003 \mathrm{~g}-i$ isochrone are counted. The background level was measured using an annulus with $2.0<r<3.0$.

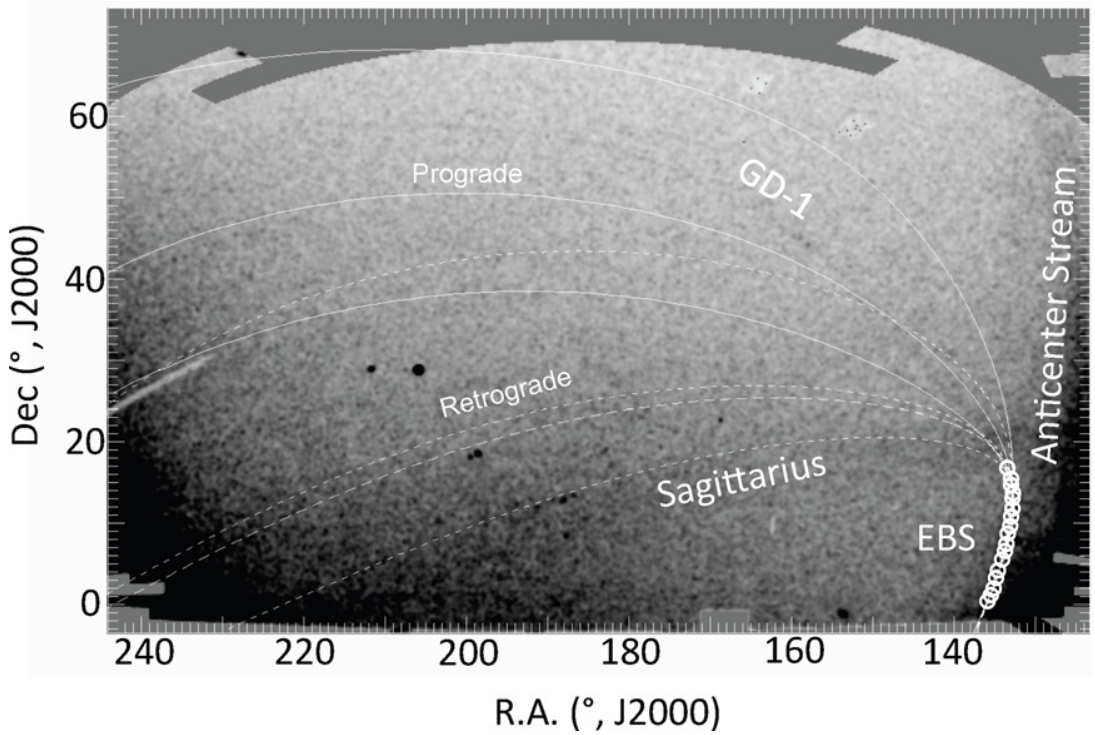

Figure 7. Best-fit orbits for EBS projected onto the SDSS DR7 footprint. Open circles indicate the normal points used to trace the stream and constrain the fit. The solid lines show the best-fitting prograde orbit, along with the $90 \%$ confidence limits. The short-dashed lines similarly show the best-fit and $90 \%$ limits on the retrograde orbit. The long-dashed line shows the best fit to the data if no velocity constraints are imposed.

their ECHOS were dwarf spheroidal galaxies. However, based on the orbit constraints below, the high-velocity dispersions of EBS and Hydra I may be due to significant heating by encounters with the dark matter subhalos (Carlberg 2009) or massive star clusters or molecular clouds in the disk.

\subsection{Constraints on the Orbit}

Mindful of the fact that tidal streams do not precisely trace the orbits of their progenitors (Odenkirchen et al. 2009; Eyre \& Binney 2009, 2011), we nevertheless estimate the orbit of the stream stars to determine whether the EBS might be related to any of the other streams in Figure 1. We do this using the Galactic model of Allen \& Santillan (1991) to compute trial orbit integrations, and matching these orbit integrations with the measured positions, distances, and velocities of the stream in a least-squares sense. We integrate orbits over a grid of possible radial velocities and proper motions, using the IDL AMOEBA downhill simplex procedure to find the minimum $\chi^{2}$ at each grid point. The grid points are separated by $1 \mathrm{~km} \mathrm{~s}^{-1}$ in radial velocity, and $0.02 \mathrm{mas} \mathrm{yr}^{-1}$ in each component of proper motion.

We fit to 17 normal points chosen to lie along the estimated centerline of the stream. We use a solar Galactocentric distance of $8.5 \mathrm{kpc}$, and stream distances and velocities as given above. We adopt velocity uncertainties for the measurements at B-7/PCI-8/PCII-20 and B-8/PCI-9/PCII-21 of $3 \mathrm{~km} \mathrm{~s}^{-1}$ (K. C. Schlaufman (2011, private communication)), positional uncertainties of 0.2 , and distance uncertainties as given above. We have attempted to measure the proper motions along the stream using the proper motions provided in DR7 (Munn et al. 2004, 2008). Unfortunately, the relatively large uncertainties and severe contamination by field stars conspire to wash out any obvious signal due to the stream. We consequently leave 
the proper motions as free parameters in the fit. Tighter constrains on the orbit will have to await the acquisition of more accurate proper motions with GAIA or the Large Synoptic Survey Telescope.

Figure 7 shows the normal points used to fit the positions along the EBS stream. Also shown are projections of the bestfit orbits on the SDSS DR7 footprint. Both the prograde and retrograde orbit models predict that the stream passes between 1 and $2 \mathrm{kpc}$ of the current position of the Sun, near the north Galactic pole in projection. At this distance, the stream would be more than $5^{\circ}$ across, and moving between three and four times faster than at apogalacticon. If the stellar stream extends along these portions of the orbits, and if the number of stars stripped from the progenitor per unit time was roughly constant over the lifetime of the stream, then we would expect the surface density near the north Galactic pole to be $\sim 15-20$ times less than it is in Figure 1. Since we expect the rate of tidal stripping to accelerate as the mass of the progenitor is diminished over time, the relative number of stream stars we might expect to see far from the progenitor would be reduced further still. If the orbit is retrograde, then the star count signal of the EBS would likely be buried within the much more populous Sagittarius stream. If there are EBS stars passing near the Sun, they will more easily be found in velocity and proper motion surveys (e.g., RAVE, GAIA, and LSST).

Figure 8 shows orbit integrations that correspond to the bestfit parameters for both prograde and retrograde models. While the formal $\chi^{2}$ for the retrograde model is $15 \%$ less than that for the prograde model, the disagreement between the predicted velocity gradient along the stream $\left(3.1 \mathrm{~km} \mathrm{~s}^{-1} \mathrm{deg}^{-1}\right)$ and the measurements at B-7/PCI-8/PCII-20 and B-8/PCI-9/PCII-21 $\left(-4.8 \mathrm{~km} \mathrm{~s}^{-1} \mathrm{deg}^{-1}\right)$ is somewhat larger (and of the opposite sign) than for the prograde model $\left(-10.7 \mathrm{~km} \mathrm{~s}^{-1} \mathrm{deg}^{-1}\right)$. Additional velocity measurements at different positions along the stream and/or proper motion measurements will be required to resolve the ambiguity. The proper motions predicted at the position of Hydra I are $\mu_{\alpha} \cos \delta=-0.15$ mas $\mathrm{yr}^{-1}$, $\mu_{\delta}=-2.67$ mas $\mathrm{yr}^{-1}$ for the prograde orbit, and $\mu_{\alpha} \cos \delta=$

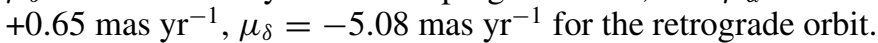
These values are of the same magnitude or less than the typical DR7 uncertainties ( $\approx 4$ mas yr -1$)$, so it is perhaps not surprising that we have been unable to identify a clear stream signature in the proper motion data.

The prograde orbit model predicts apogalacticon $R_{A}=$ $16.5 \pm 0.1 \mathrm{kpc}$, perigalacticon $R_{P}=3.0_{-0.3}^{+0.7} \mathrm{kpc}$, eccentricity $\epsilon=0.69_{-0.05}^{+0.02}$, and inclination $i=17^{\circ} \pm 0.4$, where the uncertainties correspond to the $90 \%$ joint confidence interval. For the retrograde orbit, the parameters are $R_{A}=17.7 \pm$ $0.4 \mathrm{kpc}, R_{P}=1.8_{-0.2}^{+0.4} \mathrm{kpc}, \epsilon=0.8_{-0.03}^{+0.02}$, and $i=19.9 \pm 0.7$. In either case, the EBS appears to be on a fairly eccentric orbit. This eccentricity is forced entirely by the curvature of the stream at its northern end. If we give zero weight to the B-7/PCI8/PCII-20 and B-8/PCI-9/PCII-21 velocities, the best-fitting orbit still predicts an eccentricity of $\epsilon \approx 0.8$. The stream is evidently not associated with either the ACS or the Monoceros Ring, both of which have been determined to be on very nearly circular orbits (Penarrubia et al. 2005; Grillmair et al. 2008). The combination of inclination and eccentricity takes the stream into the inner, non-spherical part of the Galactic potential, where no component of angular momentum is conserved and we see an interesting box orbit as a consequence. Frequent, oblique passages through the disk would presumably increase the potential for encounters with massive structures such as

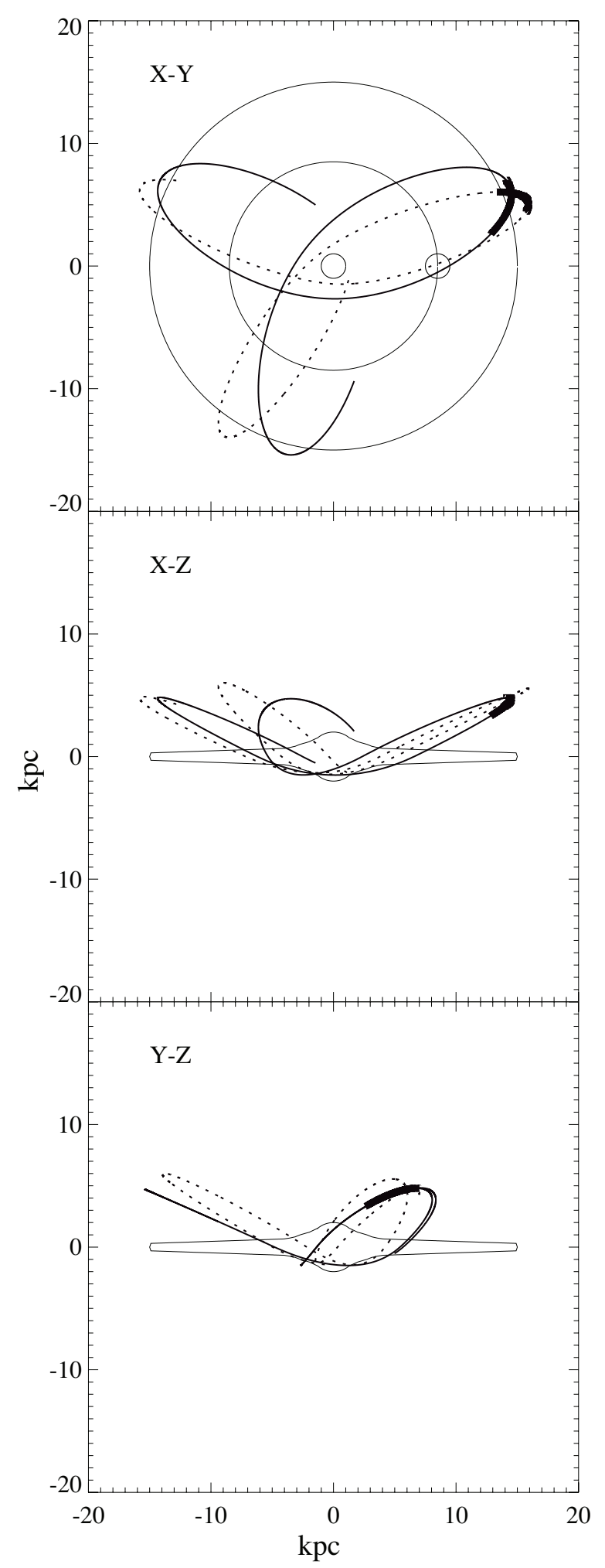

Figure 8. Best-fit orbit projections for EBS in X, Y, and Z Galactic coordinates. The heavy lines show the portions of the orbit with $-2^{\circ}<\delta<16^{\circ}$. The thin solid curve shows the best-fit prograde orbit, while the dotted curve shows the retrograde orbit that best fits all the data. The Sun's location at $(\mathrm{X}, \mathrm{Y}, \mathrm{Z})=$ $(8.5,0,0) \mathrm{kpc}$ is indicated.

stellar clusters or giant molecular clouds and may be partly or wholly responsible for the high-velocity dispersion observed in the Hydra I and the EBS stream. 


\section{CONCLUSION}

The EBS stream adds to the growing list of halo streams that can be mapped over a sufficient extent that, with suitable followup observations, they could be used as probes of the Galactic potential. A preliminary orbit estimate shows that the EBS is unrelated to either the Anticenter or Monoceros streams. The somewhat intermediate breadth of the stream together with its relatively high-velocity dispersion suggests the possibility that the progenitor could have been more massive than the globular clusters thought to be responsible for the half dozen very cold streams discovered in the SDSS footprint to date. However, if the progenitor had been a dark matter dominated dwarf galaxy, it would be difficult to understand how it could have held onto its dark matter envelope for any length of time in such a confined and eccentric orbit. On the other hand, this very orbit may have subjected both the progenitor and the stream to significant heating through encounters with massive structures in the disk.

If Hydra I is indeed the progenitor of the EBS, then it is only the second probably unbound progenitor to be associated with a tidal stream. A more detailed examination of the structure and stellar kinematics in this remnant may shed new light on the end stage of tidal disruption. Though contamination by field stars is high, Hydra I may be particularly attractive in this respect as it is four times closer to us than Bootes III (Grillmair 2009).

Refinement of the orbit will require radial velocity and proper motion measurements of carefully selected stars along the length of the stream. Given the very low surface density of stream stars and very high field star contamination, this will necessarily be an ongoing task. In this respect, the EBS may be particularly well situated for follow-up by the upcoming spectroscopic LAMOST survey. GAIA and LSST proper motion measurements may also helps us to refine the orbit and perhaps trace the stream over a much longer arc.

The author is grateful to an anonymous referee for constructive and insightful comments. Thanks also go to Kevin Schlaufman for providing the positions of ECHOS member stars. Funding for the creation and distribution of the SDSS Archive has been provided by the Alfred P. Sloan Foundation, the Participating Institutions, the National Aeronautics and Space Administration, the National Science Foundation, the U.S. Department of Energy, the Japanese Monbukagakusho, and the Max Planck Society.

Facility: Sloan

\section{REFERENCES}

Abazajian, K. N., Adelman-McCarthy, J. K., Agüeros, M. A., et al. 2009, ApJS, 182,543
Allen, C., \& Santillan, A. 1991, RevMexAA, 22, 255

Belokurov, V., Evans, N. W., Irwin, M. J., Hewett, P. C., \& Wilkinson, M. I. 2006a, ApJ, 637, 29

Belokurov, V., Evans, N. W., Irwin, M. J., et al. 2007, ApJ, 658, 337

Belokurov, V., Zucker, D. B., Evans, N. W., et al. 2006b, ApJ, 642, 137

Carlberg, R. G. 2009, ApJ, 705, L223

Carlin, J. R., Casetti-Dinescu, D. I., Grillmair, C. J., Majewski, S. R., \& Girard, T. M. 2010, ApJ, 725, 2290

Combes, F., Leon, S., \& Meylan, G. 1999, A\&A, 352, 149

de Marchi, G. 1999, AJ, 117, 303

Eyre, A., \& Binney, J. 2009, MNRAS, 399, 160

Eyre, A., \& Binney, J. 2011, MNRAS, 413, 1852

Grillmair, C. J. 1992, PhD thesis, Australian National University

Grillmair, C. J. 2006a, ApJ, 645, 37

Grillmair, C. J. 2006b, ApJ, 651, 29

Grillmair, C. J. 2009, ApJ, 693, 1118

Grillmair, C. J. 2010, in Galaxies and Their Masks, ed. D. Block, K. C. Freeman, \& I. Puerari (New York: Springer), 247

Grillmair, C. J., Carlin, J. L, \& Majewski, S. R. 2008, ApJ, 689, L117

Grillmair, C. J., \& Dionatos, O. 2006a, ApJ, 641, 37

Grillmair, C. J., \& Dionatos, O. 2006b, ApJ, 643, 17

Grillmair, C. J., \& Johnson, R. 2006, ApJ, 639, 17

Girardi, L., Williams, B. F., Gilbert, K. M., et al. 2010, ApJ, 724, 1030

Helmi, A., White, S. D. M., de Zeeuw, P. T., \& Zhao, H. 1999, Nature, 402, 53

Johnston, K. V., Spergel, D. N., \& Hernquist, L. 1995, ApJ, 451, 598

Koposov, S. E., Rix, H.-W., \& Hogg, D. W. 2010, ApJ, 712, 260

Law, D. R., Majewski, S. R., \& Johnston, K. V. 2009, ApJ, 703, 67

Majewski, S. R., Skrutskie, M. F., Weinberg, M. D., \& Ostheimer, J. C. 2003, ApJ, 599, 1082

Marigo, P., Girardi, L, Bressan, A., Groenewegen, M. A. T., Silva, L., \& Granato, G. L. 2008, A\&A, 482, 883

Martin, N. F., De Jong, J. T. A., \& Rix, H.-W. 2008, ApJ, 684, 1075

Martinez-Delgado, D., Gomez-Flechoso, M., Aparicio, A., \& Carrera, R. 2004, ApJ, 601, 242

Munn, J. A., Monet, D. G., Levine, S. E., et al. 2004, AJ, 127, 3034

Munn, J. A., Monet, D. G., Levine, S. E., et al. 2008, AJ, 136, 895

Murali, C., \& Dubinski, J. 1999, AJ, 118, 911

Newberg, H. J., Willett, B. A., Yanny, B., \& Xu, Y. 2010, ApJ, 711, 32

Newberg, H. J., Yanny, B., Rockosi, C., et al. 2002, ApJ, 569, 245

Odenkirchen, M., Grebel, E. K., Dehnen, W., et al. 2003, AJ, 126, 2385

Odenkirchen, M., Grebel, E. K., Kayser, A., Rix, H.-W., \& Dehnen, W. 2009, AJ, 137,3378

Penarrubia, J., Matiniez-Delgado, D., Rix, H.-W., et al. 2005, ApJ, 626, 128

Rockosi, C. M., Odenkirchen, M., Grebel, E. K., et al. 2002, AJ, 124, 349

Schlafly, E. F., \& Finkbeiner, D. P. 2011, submitted

Schlaufman, K. C., Rockosi, C. M., Allende Prieto, C., et al. 2009, ApJ, 703, 2177

Schlaufman, K. C., Rockosi, C. M., Lee, Y. S., Beers, T. C., \& Prieto, C. A. 2011, ApJ, 734, 49

Schlegel, D. J., Finkbeiner, D. P., \& Davis, M. 1998, ApJ, 500, 525

Smith, M. C., Evans, N. W., Belokurov, V., et al. 2009, MNRAS, 399, 1223

Willett, B. A., Newberg, H. J., Zhang, H., Yanny, B., \& Beers, T. 2009, ApJ, 697, 207

Williams, M. E., Steinmetz, M., Sharma, S., et al. 2011, ApJ, 728, 102

Willman, B., Dalcanton, J. J., Martinez-Delgado, D., et al. 2005, ApJ, 626, 85

Yanny, B., Newberg, H. J., Grebel, E. K., et al. 2003, ApJ, 588, 824

Yoon, J. H., Johnston, K. V., \& Hogg, D. W. 2010, ApJ, 731, 58

Zucker, D. B., Belokurov, V., Evans, N. W., et al. 2006a, ApJ, 643, L103

Zucker, D. B., Belokurov, V., Evans, N. W., et al. 2006b, ApJ, 650, L41 